\title{
NLTE Analysis of Central Stars of Highly Excited Planetary Nebulae
}

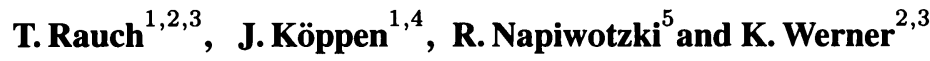 \\ ${ }^{1}$ Institut für Astronomie und Astrophysik, Universität Kiel; \\ ${ }^{2}$ Lehrstuhl Astrophysik, Universität Potsdam; \\ ${ }^{3}$ Institut für Astronomie und Astrophysik, Universität Tübingen; \\ ${ }^{4}$ Observatoire Astronomique, Strasbourg; ${ }^{5}$ Dr.-Remeis-Sternwarte Bamberg
}

Very hot central stars (CSPN) of highly excited planetary nebulae (PN) display directly the formation of white dwarfs. Only a few of these CSPN have been analyzed so far due to their low brightness and thus, the interpretation of their evolutionary status is hampered by statistical incompleteness. In the last decade many spectral analyses of very hot postAGB stars by means of state-of-the-art NLTE model atmospheres have been performed (e.g. Rauch et al. 1996; Werner \& Rauch 1994; Rauch \& Werner 1995) and our picture of post-AGB evolution has been improved.

In order to analyze some of the fainter CSPN, we selected southern CSPN $\left(m_{\mathrm{V}}>16\right)$ of highly excited PN with diameters of 18-180" (Acker et al. 1992). Images (H $\alpha$ and [O III] $\lambda 5007 \AA$ ) as well as medium resolution spectra had been taken in two observation campaigns in February and June 1996 at ESO with the 3.6m telescope. The targets and some preliminary results of our analysis are summarized in Tab. 1. A more precise analysis will be presented elsewhere.

Tab. 1. List of our targets. a: amorphous, A: ansae, (p)C: (perfectly) circular, H: central hole, J: jets, L: lobes, M: multiple shells, N: non-circular, WE: west-east

\begin{tabular}{|c|c|c|c|c|c|c|}
\hline \multirow{2}{*}{ name } & \multicolumn{2}{|c|}{ PN } & \multicolumn{3}{|c|}{ CSPN } & \multirow[b]{2}{*}{$\mathrm{He} / \mathrm{H}$} \\
\hline & $d_{\mathrm{WE}} f^{\prime \prime}$ & shape & $m_{\mathrm{V}}$ & $T_{\text {eff }} / \mathrm{kK}$ & $\log g$ (cgs) & \\
\hline PN G214.9+07.8 & 96 & $\mathrm{pCH}$ & 16.6 & 100 & 6.0 & $\odot$ \\
\hline PN G231.8+04.1 & 250 & CHM & 18.0 & 100 & 6.5 & $\odot$ \\
\hline PN G249.3-05.4 & 69 & $\mathrm{CH}$ & 18.5 & \multicolumn{3}{|c|}{ — no spectrum available _ } \\
\hline PN G253.5+10.7 & 147 & AEJH & 16.6 & \multicolumn{3}{|c|}{ - binary - } \\
\hline PN G257.5+00.6 & $>500$ & $\mathbf{a}$ & 18.0 & 100 & 5.0 & $<\odot$ \\
\hline PN G277.1-03.8 & 239 & LN & 16.5 & 100 & 5.0 & $<\odot$ \\
\hline PN G283.6+25.3 & 199 & CL & 17.4 & 100 & 7.0 & $\odot$ \\
\hline PN G293.6+10.9 & 94 & $\mathrm{CL}$ & 18.0 & 100 & 7.0 & $\odot$ \\
\hline PN G324.1+09.0 & 24 & $\mathrm{C}$ & 17.1 & \multicolumn{3}{|c|}{ — no spectrum available _ } \\
\hline
\end{tabular}

\section{REFERENCES}

Acker A., Ochsenbein F., Tylenda R., Marcout J., Schohn C. 1992, Strasbourg-ESO Catalogue of galactic planetary nebulae

Rauch T., Werner K. 1995, in: White Dwarfs, eds. D. Koester, K. Werner. Lecture Notes in Physics 443, Springer, Berlin, p. 186

Rauch T., Köppen J., Werner K. 1996, A\&A 310, 613

Werner, K., Rauch, T. 1994, A\&A 284, L5 